\title{
When clinical trials compete: prioritising study recruitment
}

\author{
Luke Gelinas, ${ }^{1}$ Holly Fernandez Lynch, ${ }^{2}$ Barbara E Bierer, ${ }^{3}$ I Glenn Cohen ${ }^{1}$
}

${ }^{1}$ Petrie-Flom Center at Harvard Law School and Harvard Catalyst, Cambridge, Massachusetts, USA ${ }^{2}$ Petrie-Flom Center at Harvard Law School, Harvard Catalyst, and Center for Bioethics, Harvard Medical School, Cambridge, Massachusetts, USA ${ }^{3}$ Brigham and Women's Hospital, Harvard Medical School, and Harvard Catalyst, Boston, Massachusetts, USA

Correspondence to Dr. Luke Gelinas Petrie-Flom Center Harvard Law School, 23 Everett Street, Cambridge, MA 02138

Received 11 May 2016 Revised 14 July 2016 Accepted 12 November 2016 Published Online First 20 January 2017

\section{Linked}

- http://dx.doi.org/10.1136/ medethics-2016-103998 - http://dx.doi.org/10.1136/ medethics-2016-103999 - http://dx.doi.org/10.1136/ medethics-2016-104000 - http://dx.doi.org/10.1136/ medethics-2017-104165

\section{CrossMark}

\section{ABSTRACT}

It is not uncommon for multiple clinical trials at the same institution to recruit concurrently from the same patient population. When the relevant pool of patients is limited, as it often is, trials essentially compete for participants. There is evidence that such a competition is a predictor of low study accrual, with increased competition tied to increased recruitment shortfalls. But there is no consensus on what steps, if any, institutions should take to approach this issue. In this article, we argue that an institutional policy that prioritises some trials for recruitment ahead of others is ethically permissible and indeed prima facie preferable to alternative means of addressing recruitment competition. We motivate this view by appeal to the ethical importance of minimising the number of studies that begin but do not complete, thereby exposing their participants to unnecessary risks and burdens in the process. We then argue that a policy of prioritisation can be fair to relevant stakeholders, including participants, investigators and funders. Finally, by way of encouraging and helping to frame future debate, we propose some questions that would need to be addressed when identifying substantive ethical criteria for prioritising between studies.

\section{INTRODUCTION}

Imagine that at a particular cancer centre there are five ongoing oncology trials intended for the same patient population, each needing to recruit at least 100 participants to achieve adequate statistical power, and each seeking to reach its recruitment goals this year. Suppose, further, that there are 400 patients who will be available to be recruited at the site, all of whom are exchangeable between trials; each patient meets the inclusion criteria for any of the five trials, but can only participate in one (because of exclusion criteria for the others, including participation in other trials and time). If all five trials recruit simultaneously with no priority between them, it will be impossible for all of them to meet their recruitment targets. Moreover, it is possible in this situation that none will achieve adequate statistical power, leading to wasted resources, inappropriate risks and burdens to participants who were enrolled and delay in generating data critical to improved patient care.

Situations like this are not merely hypothetical. Cancer centres, in fact, routinely host competing clinical trials and competing biomedical studies are present in other clinical contexts as well, such as stroke treatment and emergency medicine. ${ }^{1-5}$ Moreover, there is good evidence that the actual level of competition between trials is a predictor of low accrual, with increased competition unsurprisingly tied to increased recruitment shortfalls. ${ }^{1}$

Recruitment competition raises a number of broadly ethical issues, perhaps the most pressing of which is how to go about determining which patients should be offered enrolment in which trials on which timelines or indeed whether institutions should interfere in this process at all. There are at least four ways institutions might approach this question.

First, some argue that clinicians and investigators have a duty to disclose all open trials at a given institution (and perhaps beyond) for which individuals are eligible in order to enable them to make an informed choice about which one to join. ${ }^{6}{ }^{i} \mathrm{~A}$ second approach denies that there is any obligation to offer potential participants a choice between competing trials and instead leaves the choice up to clinicians, who typically offer trials based on their own estimation of a trial's therapeutic potential or other, more idiosyncratic factors (such as the level of investment of the clinician or department chief in different studies and what they have to gain from prioritising some over others). ${ }^{2}$ A third option, motivated by avoiding bias and ensuring scientific integrity, is to employ a randomising procedure to decide which trials to offer individuals, similar to the use of randomisation to allocate participants to different arms within individual studies. ${ }^{4}$ Finally, the fourth approach is for institutions intentionally to prioritise studies for recruitment (eg, by declining to let some studies open if doing so would cause demand for patients to substantially exceed the supply), allowing some trials to recruit ahead of others and thereby limiting the number of trials that compete for the same potential participants at a given time.

In this article, we argue that the fourth approach, intentional prioritisation, is ethically acceptable and indeed prima facie preferable to other alternatives. We proceed as follows. In Section 'The inevitability of allocation', we suggest that situations in which clinical trials compete for limited participants are best construed as analogous to rationing scenarios where a scarce resource (here, trial participants) must be allocated in acceptable ways. In this light, the question is not whether we should allocate participants between trials-there is no avoiding that-but rather how best to do so. In Section 'Recruitment competition and the problem of

${ }^{\mathrm{i}} \mathrm{A}$ recent survey suggests that this is not common practice at stroke centres (at least) running more than one clinical trial: $75 \%$ of respondents at 24 StrokeNet sites reported that they do not inform potential participants of all available trial options at their institution (see ref. 3 . 
non-completion', we point out that recruitment competition can be the cause of recruitment shortfalls in studies that begin but do not recruit enough participants to achieve adequate statistical power and that, in the process, expose participants to unjustified risks and burdens-which we have strong ethical reason to avoid. We then propose that the best way to address this problem is via a policy of intentional prioritisation that minimises the number of trials recruiting concurrently from the same population, thereby making non-completing studies less likely. In Section 'Is prioritisation fair to relevant stakeholders?', we argue that, in addition, an institutional policy of prioritisation can demonstrate adequate consideration for the rights and interests of relevant stakeholders, in particular, participants, investigators and funders. In Section 'Prioritising studies', we reflect on how to approach decisions about which trials to prioritise ahead of others, which we hope will spur and help frame future debates, but which is by no means meant to be exhaustive or conclusive. We conclude by summarising our argument and issuing a call for engagement and vigorous debate in the research community over the issues raised by prioritisation.

\section{THE INEVITABILITY OF ALLOCATION}

The example at the start illustrates a classic dilemma in medicine and indeed social policy generally: how to allocate a scarce and valuable resource-in this case, potential trial participants-in acceptable ways when there is not enough to go around. In the medical context, familiar examples include the allocation of transplant organs, of influenza vaccine in a pandemic and of intensive care unit (ICU) beds when oversubscribed. There are, however, important differences when rationing potential trial participants. Most importantly, unlike ICU beds or kidneys, trial participants are individual human beings with interests that deserve consideration and rights that must be respected in allocation decisions (see below; Section 'Is prioritisation fair to relevant stakeholders?'). ${ }^{\text {ii }}$

Nonetheless, we cannot avoid taking a position on how a limited pool of participants should be allocated between competing trials, since in all cases participants are indeed allocated, just according to different methodologies. Consider the approaches in the literature described earlier. An institutional policy of letting patients choose between trials is one mechanism for allocating participants; a policy of letting clinicians decide which trials to offer which patients is a second form of allocation and a policy of randomising between competing trials is a third. While it is true that these approaches leave room for individual discretion and choice (either the patient's or the clinician's) or chance (randomisation), such features do not render them any less of an allocation scheme. Moreover, even when an institution has no official intentional allocation policy, adopting instead a laissez-faire approach to competition between trials, the resulting system will itself, in practice, rest on and embody particular allocation principles. For example, an institutional hands-off approach to competing trials may implicitly rest on the principle of ability-to-pay, on the assumption that better

\footnotetext{
${ }^{i i} \mathrm{~A}$ related difference is that, unlike kidneys or ICU beds, potential participants can decide not to be allocated at all, by declining to participate in research and accepting the standard of care available outside the study. This would prove relevant to the argument that follows if different methods for allocating trial participants influenced the absolute number of people willing to take part in research (see Section 'Is prioritisation fair to participants?').
}

funded studies with more money to spend on recruitment will typically have better chances of meeting recruitment targets. ${ }^{\mathrm{iii}}$

How, then, should potential participants be allocated between trials? Our answer, which will emerge over the next three sections, is that allocation should be determined by an institutional policy that purposefully allows some studies to recruit ahead of others. Such a prioritisation approach is motivated in large part by its ability to mitigate concerns over what we call 'the problem of non-completion'.

\section{RECRUITMENT COMPETITION AND THE PROBLEM OF NON-COMPLETION}

Consider again the example at the start. In this situation, we have strong ethical reason to avoid the following scenario: all five trials enrol some participants and begin the protocol, exposing their participants to risk in the process, but due to recruitment competition none of them recruit enough participants to be adequately powered. Likewise, assuming all else is equal between the trials, we have only slightly less strong reason to avoid a scenario where all five trials enrol some participants and begin the research but only one of them enrols enough to be adequately powered. Generalising from this, we have strong ethical reason and a pro tanto obligation to minimise the number of trials that begin but do not complete.

This obligation is grounded in a fundamental tenet of research ethics and reflected in its history and canonical texts, which since Nuremberg have recognised that clinical research must be justified by an appropriate risk-benefit ratio. The ethical justification for exposing individuals to the risks and burdens of clinical research is the social value of the 'generalisable' knowledge it stands to produce. ${ }^{\text {iv }}$ While clinical trials sometimes benefit individual participants, they are not ethically justified by (or merely by) this fact, but rather primarily by appeal to the potential treatment advances made possible by the knowledge gained from such research. ${ }^{\mathrm{v}}$

Returning to our example, the main ethical concern with trials that begin but do not recruit enough participants to be adequately powered is that they will be unable to generate the sort of conclusions that form the basis of socially valuable knowledge. In addition to the waste involved with such trials, the main ethical worry is that participants will be exposed to risks and burdens for no compensating social good, leaving those risks and burdens in danger of being unnecessary and unjustified. Since we have strong ethical reason to avoid exposing participants to unjustified risks and burdens, we have strong ethical reasons to minimise the number of trials that begin but do not complete.

Moreover, informing participants of the possibility of non-completion-and the possibility that their acceptance of risks and burdens may be for naught-as some suggest, ${ }^{15} 16$ does not completely remove the ethical concerns over

\footnotetext{
iiiStudies with enough resources to hire staff to recruit in-person, for example, are likely to be at an advantage here (see eg, ref. 7). It is worth noting that the principle of ability-to-pay is often seen as one of the least ethically defensible rationing principles. ${ }^{8-10}$

iv $S e e$, for example, the US 'Common Rule' governing research with humans at 45 Code of Federal Regulations (CFR) 46.111.

${ }^{\mathrm{v}}$ The requirement of social value may also be justified in part by appeal to preventing wasted resources. ${ }^{11}$ Although we choose to focus on the way in which incomplete studies expose participants to unnecessary risks, the issue of waste should not be underplayed and can itself help to motivate a prioritisation approach (see refs 12-14).
} 
non-completing studies. This is because there is a duty to ensure participants are not subject to unjustified risks independent of the duty to respect participant autonomy via informed consent. ${ }^{11}$ Even if participants understand the possibility of non-completion and consent to this risk-that is, even if their autonomy is fully respected-they can still be harmed and wronged by being exposed to risks and burdens unnecessarily. Indeed, this is precisely the foundation for the ethical and regulatory requirement to minimise risks to research participants.

In some cases, recruitment shortfalls will be unpredictable and unforeseeable, such that there is little we can do to prevent or minimise the chance of studies beginning but not completing. However, this is not always the case. In many cases, institutions know (or should know), based on data and past experience, that there are unlikely to be enough volunteers to adequately power all the research it would like to see done in a particular sphere. In these cases, institutions can (and should) also realise that the more studies that compete for this limited pool of participants, the greater the likelihood that one or more of them will begin but not complete, and thus the greater the likelihood that participants will be exposed to unnecessary risks.

\section{Minimising non-completion by prioritising}

But what exactly should institutions do under these conditions? Our answer is that institutions should minimise recruitment competition by prioritising some studies for recruitment over others. There are at least two ways institutions might do this. First, institutions could limit the number of competing studies open at once, allowing some trials to open and begin recruiting ahead of others and giving those prioritised enough time (within reason) to enrol enough participants to be adequately powered, before permitting more competing studies to open. Second, rather than preventing some trials from opening, institutions could impose a rank list ordering system on already open trials, allowing the trials at the top of the list to approach patients first and subsequent, competing trials to offer enrolment only when individuals decline to enter studies ranked higher on the list. The upshot of either strategy would be to minimise the number of studies recruiting concurrently from the same population. ${ }^{\mathrm{vi}}$

The further practical details of a prioritisation model might vary from institution to institution and depend on the particulars of the situation. Because the fewer the studies, the less the competition, permitting only one study to recruit at a time would be safest in terms of satisfying the duty to minimise noncompleting studies. But in some cases, limiting the number of recruiting trials to only one will be unnecessarily restrictivenamely, whenever we have good reason to think that there will be enough participants to power multiple, if not all, studies. Limiting the number of studies to one may also be infeasible or even ethically questionable, as might happen if two competing studies cannot be appropriately compared for prioritisation purposes; in that case, there may be good reason to give potential participants a choice between them. For example, imagine that one trial is high risk but high reward (eg, there is a good chance of dying from the treatment, but if you don't die, you might be cured), while a second trial with the same inclusion/exclusion criteria as the first is low risk but lower reward (eg, the interventional agent may put the disease in remission, but will definitely not cure it). Given the radical differences between these trials in

\footnotetext{
${ }^{\mathrm{vi}}$ We also note that these two strategies may be complementary and
} profitably used together in certain circumstances. terms of their endpoints and risks/benefits, institutions may be on firmer ethical ground letting patients choose than delaying one from recruiting. ${ }^{\text {vii }}$ In general, the number of studies that institutions permit to compete for recruitment should be based on a careful assessment of how many participants are likely to be available over a particular time frame and location, as well as an assessment of the comparability of trials, which will vary from situation to situation.

By minimising the number of studies competing for the same participants, the prioritisation model aims to reduce the number of research participants exposed to risk unnecessarily in underpowered studies. This gives it an important advantage over competing approaches. Allowing patients or clinicians to choose between trials, as some advocate, ${ }^{2}{ }^{6}$ will not address or minimise the problem of competing studies, since patients and clinicians are not responsible for the completion of studies and will (and perhaps should) base their choice on considerations other than making sure that the institution's prioritised trials recruit enough to complete. Likewise, randomising participants between competing studies (as per ref. 4) does not address, and indeed may exacerbate, the problem. This is because if participants are blindly randomised between competing studies, and if the allocation is truly random (ie, if the ex ante likelihood of winding up in each of the relevant studies is equal), we should expect each competing study to receive roughly the same number of participants. But if there are not enough participants to satisfy the statistical needs of each of the studies (as in our original example), then all studies may obtain too few participants. In these situations, distributing participants evenly across competing studies would maximise rather than minimise the number of non-completing studies. Thus, prioritisation is the best of these approaches for avoiding the problem of non-completion.

\section{IS PRIORITISATION FAIR TO RELEVANT STAKEHOLDERS?}

We must still address a fundamental set of questions involving whether prioritisation can be fair to relevant stakeholders. When evaluating this question, we will assume that, if institutions were to adopt a policy of prioritisation, they would be constrained by ethically defensible criteria-principles that are not obviously unfair and on which reasonable people might agree-when deciding which trials should be prioritised ahead of others (see Section 'Prioritising studies'). In this section, we focus on whether a policy of prioritisation would be sufficiently respectful of the rights and interests of three parties: participants, investigators and trial sponsors.

\section{Is prioritisation fair to participants?}

While a policy of prioritisation can minimise the number of people exposed to risks and burdens unnecessarily, and would to that extent be salutary for participants enrolled in clinical trials, it might nonetheless show inadequate consideration for participant rights and interests by limiting the number and/or type of clinical trials available to them.

We do not believe that individuals have a positive moral (or legal) right to have all the trials that could be offered by an institution available to them, that is, a right to dictate that institutions open certain studies at certain times or make all open studies equally available (rather than, as on a rank list approach,

\footnotetext{
vii The more ideal approach in this context would be to facilitate the opening of one of the trials at a different site, although that may be beyond an institution's control.
} 
offering prioritised studies first), as this would take participant autonomy to the extreme. Even the growing and welcome trend of participant engagement falls short of suggesting that institutions should be forced to surrender control over their institutional mission and selected priorities in this way. It is, however, important to note that this issue is distinct from whether patients have a right to be offered all trials that are prioritised (assuming there are more than one) and currently recruiting and for which they are eligible. To our eyes, patients will at least have a right to ask clinicians which options are available to them, and clinicians will have a duty at least to recommend the prioritised trial they think will be best for their patients, though this is a topic that would require further discussion. ${ }^{\text {viii }}$

Even if individuals do not have a positive right requiring institutions to open or offer certain trials at certain times, might they have a legitimate interest in this, one that carries ethical weight? While we must take care to avoid a therapeutic misconception-that is, the assumption that participants will in fact benefit from clinical trials ${ }^{17}$ - the fact that some trials do hold the prospect of direct benefit, sometimes for very sick people with few treatment options, and the chance that a prioritisation approach may result in fewer options for these patients, demands that this objection be addressed.

There are two versions of the basic concern. The first points out that particular individuals may be better-off in studies that the institution delays from recruiting under a policy of prioritisation (and which would be available to them under a different approach) and argues that these individuals may have a justifiable complaint. The problem with this line of thought is that any institutional policy for allocating participants or determining which trials to offer at which times may result in some patient health interests being left unsatisfied, just as any way of allocating a scarce influenza vaccine, or ICU beds or kidneys, will not provide the item to everyone who could benefit from it. ${ }^{\mathrm{ix}}$ In all of these cases, there is the need for an independently fair policy of prioritisation, one that provides a sound ethical basis for allocating precisely when everyone's interests cannot be satisfied simultaneously.

The second version of the objection focuses not on the interests of particular individuals per se but rather on the aggregate interests of particular patient populations. Perhaps certain prioritisation approaches could inadvertently favour some patient populations over others, for example, if oncology trials were always prioritised over HIV trials. However, this is not likely to be a concern in many cases, since trials aimed at the medical concerns of different patient populations are unlikely to be competing for the same pool of potential participants. Moreover, if the studies really would be competing for the same population, considerations about which trial/s will maximise aggregate patient interests can be factored into substantive prioritisation decisions. However, it is also possible that institutional

\footnotetext{
viii On this issue, see ref. 6 . It is not clear to us that clinicians always have a duty to proactively disclose all prioritised trials for which a patient is eligible. Just as physicians have no obligation to disclose clearly inferior treatments as live options, researchers have no obligation to disclose clearly suboptimal trials that stand to benefit patients less than other trials open to them. In some situations, moreover, disclosing all options is likely to overwhelm patients and results in diminished autonomous decision-making.

${ }^{\mathrm{ix}}$ Note that this includes unrestricted competition approaches, since some patients may prefer not to choose between multiple studies, may choose trials that do not reflect their long-term interests, or, if trials do not complete due to competition, may have their preference not to be exposed to unjustified risks and burdens frustrated.
}

prioritisation (resulting in a limited choice set for potential participants) could result in some version of 'crowding out' in which more members of a patient population would be willing to participate in research if they had more choice between trials than if that choice is limited. In this context, prioritisation itself might result in a smaller pool of willing participants who might benefit from clinical trials. While we stress that this objection rests on an empirical assumption that would require support (and that we know of no such support), if it did hold, this would give institutions some reason to permit multiple competing studies, which would need to be weighed against the reasons to minimise non-completing studies when determining how to proceed.

\section{Is prioritisation fair to investigators and funders?}

Even if a policy of prioritisation shows adequate consideration for the rights and interests of participants, the burden it imposes on investigators whose research is not prioritised, and whose recruitment and trials are delayed as a result, may be problematic. For one, the self-interest of these investigators (eg, scientific reputation, career advancement) may often be set back. While these setbacks may be justifiable if they are an unintentional by-product of implementing an ethically satisfactory solution to competing trials, a deeper worry is that institutions would treat investigators unfairly and indeed wrong them by impeding their research. This concern may be especially salient in cases where studies already funded by external entities (National Institutes of Health (NIH), industry, etc) are delayed from recruiting by an institutional policy of prioritisation. In these cases, funding might, as a result of the delay, be jeopardised or withdrawn altogether by funders-in which case investigators could argue that they have been treated unfairly, given the amount of time and effort spent securing funding and the reputational risk of not delivering to the sponsor.

Relatedly, there are concerns about how a policy of prioritisation would impact the interests of funders. Entities who fund studies in accordance with ethically defensible considerations (one of which may be the ability to complete the study in a timely manner), only to see those studies delayed at the institutional level by a policy of prioritisation, might claim that the institution is impeding the ability of funders to make wellinformed funding decisions for no good reason, given that the funders themselves already take the relevant considerations into account.

In addressing these concerns, it is important to note that, in general, the appropriate criteria for deciding which studies to prioritise for recruitment are likely to align with the appropriate criteria for deciding which studies to fund. If, for example, Trial 1 has greater expected social value than Trial 2, that is a reason both for funders to sponsor Trial 1 over Trial 2 and for institutions to prioritise Trial 1 for recruitment over Trial 2. Despite this, the trials prioritised by funders and the trials prioritised by institutions are unlikely to align completely, if for no other reasons than that funders and institutions may not always agree on which prioritisation criteria should be employed and institutions may run trials from multiple funders. Given this, the question of whether institutional prioritisation can be fair to investigators and funders turns on the details of how a prioritisation system would be implemented in practice, and whether there could be sufficient coordination between relevant parties.

While a detailed implementation proposal is beyond the scope of this article, we will briefly propose three considerations that we believe can facilitate an ethical prioritisation model. We do not necessarily claim that they are the only considerations or 
sufficient by themselves. Nonetheless, their availability and plausibility does suggest that a prioritisation approach could be implemented in ways that take account of the interests and reasonable expectations of investigators and funders.

First and most basically, institutions would have a duty to proactively disclose that they will be prioritising studies for recruitment-their intent to prioritise- and to inform investigators of what the basic system will look like and involve. Among other things, this could serve to inform investigator expectations ahead of time about the types of recruitment delays they might expect, allowing them to be well informed about the personal implications of the policy to them. In the case of cooperative group, network or industry trials where funding is precommitted, being transparent about the intent to prioritise at the institutional level would also give funders the chance to proactively address priority issues as part of the negotiation with different sites. ${ }^{x}$

Second, institutions would have a duty to disclose how they will prioritise studies for recruitment-what considerations and criteria they will use in prioritising some studies ahead of others-and to engage and seek input from researchers, funders and the public over appropriate criteria. ${ }^{\mathrm{xi}}$ If the institution is transparent with investigators about the content of the prioritisation criteria, and proactive in disclosing them, investigators could make choices to bring their studies more in line with the institution's priorities-which, ideally, will include promoting the most socially valuable research. Institutions may also be obligated to take additional steps to establish fair procedures for assessing tenure and career advancement, such as giving some measure of credit to investigators who are funded but have their studies delayed by institutional prioritisation decisions.

Finally, it is common practice for institutions to subject proposals for clinical trials to a process of prereview before investigators submit them to potential funders. As part of this prereview process, institutions could require trials to be routed through a process of prioritisation review, with each protocol being evaluated and ranked on the basis of the recruitment prioritisation criteria endorsed by that institution. While such a ranking system could take various forms, the basic idea is that, the better protocols fare by the institution's prioritisation metrics - that is, the more likely they are to be prioritised for recruitment by the institution-the higher score they would earn, and vice versa. Because the institution will not know at this stage which trials will be funded or which trials will be ready to begin at which times, an ordinal ranking of studies will not be possible. Instead, institutions might assign a rough, noncomparative ranking at this phase, with (eg) a ranking of ' 1 ' meaning that a trial's importance to an institution is very high and that it is likely to be prioritised, a ' 2 ' meaning the trial's importance is slightly less high and so slightly less likely to be prioritised and so on.

Such an approach has the potential to mitigate several obstacles to implementing a prioritisation model. First, when possible, the scores assigned by the institution could and should be disclosed to investigators, allowing them to be well informed from an early stage about the likelihood that their particular

\footnotetext{
${ }^{\mathrm{x}}$ Relatedly, it will often be in the best interests of funders to hold institutions accountable for meeting recruitment targets and completion; a policy of prioritisation, worked out proactively in negotiations between sponsors and sites, may nicely inform this aspect of the relationship.

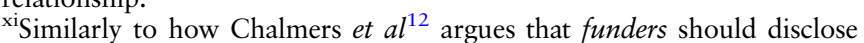
how they decide which research to support to the public and seek input
} from the public over what types of research should be prioritised. study will be prioritised or held back for recruitment. Such a ranking system would also give researchers a more concrete sense of how the prioritisation criteria (which should be transparent from the get-go) will be applied in practice.

Second, the institution's evaluation system and ranking of particular protocols could, and should, be submitted to potential funders as part of the application for funding. If potential funders knew how the institution weighs the importance of the research for prioritisation decisions, funders would be able to include this consideration in their deliberation over which studies to fund. This might promote further alignment of research priorities between institutions and funders, with sponsors having at least some additional reason (not necessarily decisive) to fund studies that score well by the institutional metric.

As mentioned above, one practical hurdle with this approach is that institutions will not generally be able to predict in advance which trials will successfully attain funding or when they will receive funding and be ready to begin. Thus, institutions will not be equipped during preapproval to decide on an actual prioritisation order between studies. Institutions might address this shortcoming by also requiring postapproval prioritisation review, after funding has been received, so that the studies actually 'up' and ready to begin can be compared with each other and prioritisation decisions finalised. This would, we acknowledge, still involve unpredictability. For example, some studies ranked highly during preapproval may be held back from recruiting if there are equally important or more important studies ready to begin (vice versa for studies ranked low during prereview). There may be an obligation on the part of funders to permit some flexibility under these circumstances, so long as the postreview decision to delay trials is based on defensible ethical considerations.

We acknowledge that, on our approach, investigators and perhaps funders as well may spend time and energy on proposals that are held back from recruiting by a policy of prioritisation and that in some cases may never open or get off the ground. However, this same risk is present whenever there is uncertainty about funding. More importantly, time and energy would be no less wasted outside a prioritisation model on trials that, due to competition for recruitment, are inadequately powered and do not complete. While the mere risk of wasted time and effort is not reason to reject a prioritisation model, high degrees of transparency and collaboration will be crucial to mitigating those risks and distributing burdens across shareholders in acceptable ways.

\section{PRIORITISING STUDIES}

Thus far, we have argued that a prioritisation model has important ethical advantages over other approaches, grounded in the ability of prioritisation to mitigate the risks of non-completing studies, and that a policy of prioritisation can show adequate consideration for the rights and interests of relevant stakeholders. Taken together, these considerations permit us to conclude that a system of prioritisation can be ethically permissible and indeed prima facie preferable to alternatives. While any prioritisation model that limits the number of concurrently recruiting trials will address the problem of non-completion that motivates our approach in this article, prioritisation systems may yet be ethically better or worse, depending on the substantive principles they use to determine priority between studies. A prioritisation model employing principles that are independently fair and promote the public good would be better, from an ethical perspective, than a model employing arbitrary principles 
or principles meant solely to maximise institutional profits, even if both of these models were equally successful at minimising non-completing studies.

For this reason, we will end the paper by asking about the content of substantive prioritisation considerations and how institutions should decide which particular trials to prioritise ahead of others. We do not endeavour to provide an answer to this question here or even to raise all the questions that would need to be asked, but rather to open a dialogue about how trials might be prioritised once we accept that this is the preferable way to address the problem of competing studies. There is a robust literature on rationing and priority setting in medical contexts that can guide us in this context. While some of the questions and considerations from that literature may not apply straightforwardly to prioritising trials, we think the following issues are relevant and demand consideration.

\section{Should all trials be treated equally?}

Potentially competing trials might be treated equally, so that each trial gets an equal chance at being prioritised for recruitment in a fair lottery process (eg, a series of fair coin tosses). That said, it is commonly argued that prioritising on the basis of random lotteries is preferable only when other, more ethical forms of allocation are unavailable. ${ }^{9}{ }^{10}$ Consider, for example, a situation where we must choose how to distribute a limited pandemic influenza vaccine. We should reject the idea that everyone ought to be given an equal chance of receiving the vaccine. At the least, we should give priority to medical workers treating the sick and working to prevent the spread of the disease in influenza-stricken areas; that is, to those who can do the most to prevent or alleviate the spread of the disease and the suffering caused by it and in so doing benefit others. ${ }^{\text {ii }}$

\section{Prioritising on the basis of social benefit?}

Analogously, rather than give each trial an equal chance of being prioritised, it may be ethically preferable to prioritise trials based on their potential to benefit the public by advancing treatment and health. While this seems prima facie appropriate, given that the aim of clinical research is to promote the public good, it raises a number of challenging issues.

For one, as the literature on medical rationing demonstrates, there are general questions about how to measure 'health benefit' and balance different kinds of benefits against each other. Is it more important to promote objective measures of health, such as increased life expectancy and cures for disease, or to promote subjective quality of life and disease management? Should institutions adopt a mixed metric of health from the economic literature on medical rationing, such as the quality-adjusted life year or disability-adjusted life year (QALY or DALY), when evaluating a trial's potential benefits? What is the optimal ratio of trials aimed at cures and trials aimed at symptom alleviation? Should the timing of treatment advances matter, with more weight being given to trials that stand to advance treatment the quickest? Should gains in treatment and health count the same regardless of who they stand to benefit or should they count for more when they have the potential to

\footnotetext{
xii Some will also hold that priority should be given to the young over the elderly, on the grounds that the young stand to lose more than the elderly if killed by the flu. But this is a more controversial position. See ref. 9 .
}

benefit the sickest or least well-off, or the young, or the most socially productive?

Even more basically, for trials to be prioritised in this way, we would need some reliable way to measure, ex ante, the prospective benefits of different studies. Because we do not know the results of clinical trials beforehand, we cannot know before the research is completed the extent to which different trials will actually advance treatment or health. While we do not think this is an insuperable obstacle-in the real world, we allocate scarce medical items (ICU beds, kidneys, etc) by their potential benefits under conditions of uncertainty all the time-careful and sustained consideration would be needed before a principle prioritising trials on the basis of social benefits could be confidently applied.

\section{Instrumental and pragmatic considerations}

There are, further, instrumental considerations that contribute indirectly to the impact a study might have and that may be ethically relevant. One important instrumental factor is the likely costs of the interventions being tested or their likely level of insurance coverage for the patients who could benefit from them. On its face, the more people who are likely to have access to a promising novel agent, the greater we can expect its positive impact to be. However, while these considerations seem relevant in theory, in practice we think that most institutions will not be in a good position to reliably assess how potential differences in the cost of novel agents impact long-term societal well-being, such that considerations of cost should not play a large role in actual prioritisation decisions.

A second instrumental consideration involves institutional interests. If Trial $\mathrm{A}$ is likely to benefit the institution more and/ or cost it less than Trial B, perhaps because the funder of Trial A will pay the institution more per participant enrolled than the funder of Study B (eg), there will be pressure on the institution to prioritise Trial A ahead of Trial B. To what extent is it legitimate for institutions to make prioritisation choices on the basis of institutional self-interest? While there are ethical limits to how much institutions can favour their own interests, the situation can be complicated by the long-term goals of the institution and the importance of success. If the institution in question is devoted to finding a cure for cancer and is attracting talented young cancer researchers, promoting its long-term interests may be very ethically important, given that this will also serve patient interests on a larger scale. A similar dynamic might also pertain in cases where prioritisation choices impact the likelihood of grant renewal.

Institutions will also need to grapple with a range of more pragmatic questions. Should trials that are open longer be given priority or should time since opening be irrelevant to determining recruitment priority? Is it acceptable for institutions to prioritise trials initiated or led by their own investigators over trials initiated elsewhere for which the institution is one (but not the primary) site? Should institutions prioritise the trials of junior investigators ahead of the trials of senior investigators or should the career stage of investigators be irrelevant to prioritisation decisions? Addressing these questions will require high degrees of transparency and sensitivity on the part of institutions.

While there are sure to be other issues that call for consideration, we hope that the preceding discussion at least gives some sense of the types of questions that would need to be asked when considering institutional prioritisation criteria. We acknowledge that the issues here are complex and difficult. 
However, we do not think they are any more intractable than other important concepts and debates in research ethics. ${ }^{\text {xii }}$ If done with sufficient care, such that the prioritisation criteria adopted by institutions are constrained by and reflect defensible ethical considerations, the resultant prioritisation system could promote more over less important research and approximate an ethically optimal mechanism for distributing participants between trials. This would give us even further reason to prefer a prioritisation model.

\section{CONCLUSION}

In this article, we have advanced and defended an undertheorised approach to competing trials, claiming that a prioritisation system according to which institutions minimise the number of competing trials by permitting some trials to recruit ahead of others has important advantages over other approaches. In particular, a system of prioritisation can reduce the number of studies that begin but, due to competition for recruitment from other studies, do not recruit enough participants to be adequately powered, in a way that gives it an advantage over other approaches. Moreover, we have also argued that a policy of prioritisation can show adequate consideration for the rights and interests of relevant stakeholders, in particular, participants, investigators and sponsors. Taken together, these considerations support the conclusion that a policy of prioritisation is ethically permissible and indeed prima facie preferable to other approaches. Finally, we have called attention to some of the issues that would need to be considered when adopting and applying substantive prioritisation criteria.

As we have said throughout, there is still much work to be done. We need more work modelling different approaches to implementing prioritisation and analysing how they bear on the interests of investigators and sponsors. Perhaps more importantly, we need a vigorous international debate over the range of acceptable substantive criteria institutions could adopt for prioritisation decisions. Despite its importance and difficulty, prioritisation has been a neglected and underdeveloped topic in the research ethics literature. We hope that we can help convince others that a prioritisation approach deserves to be taken seriously and encourage the type of sustained attention and debate it deserves.

\footnotetext{
xiiisuch as, for example, determining whether something counts as a case of 'exploitation' or 'undue inducement' in an ethically objectionable or prohibitive sense or specifying the threshold for studies to count as 'minimal risk'.
}

Acknowledgements We thank the reviewers for their thoughtful comments that helped to strengthen the arguments herein. We are also grateful to Russell Spivak and Jennifer Majersik.

Contributors All four authors meet the criteria set out in ICMJE Recommendations 2013. IGC oversaw the project, LG took the lead and spent the most in terms of time, energy, ideas, suggestions for revisions and so on, with HFL and BEB second and third on that count.

Funding National Center for Research Resources and the National Center for Advancing Translational Sciences, NIH (Award UL1 TR001102).

Competing interests None declared.

Provenance and peer review Commissioned; externally peer reviewed.

\section{REFERENCES}

1 Bennette CS, Ramsey SD, McDermott CL, et al. Predicting low accrual in the National Cancer Institute's Cooperative Group Clinical Trials. J Nat/ Cancer Inst 2015;108:pii: djv324.

2 Ibrahim GM, Chung C, Bernstein M. Competing for patients: an ethical framework for recruiting patients with brain tumors into clinical trials. J Neurooncol 2011;104:623-37.

3 Majersik J. Ethics and Bias of Enrolling in Competing Trials: A StrokeNet Survey and Status Update. http://www.nihstrokenet.org/docs/default-source/ presentations/isc-nashville-2015/majersik_nashville15.pdf?sfvrsn=2. (accessed 8 Apr 2016)

4 Saver JL. Coping with an embarrassment of riches: how stroke centers may participate in multiple, concurrent clinical stroke trials. Stroke 1995;26:1289-92.

5 Roberts J,Silbergleit R. Competing Protocols for the, Same Patient Population: Who Should Choose? NIH Clinical Center Ethics Rounds, April 1, 2015. http://videocast. nih.gov/Summary.asp?File=18926\&bhcp=1. (accessed 13 Dec 2015).

6 Gordon EJ, Micetich KC. Competing clinical trials in the same institution: ethical issues in subject selection and informed consent. IRB 2002;24:1-7.

7 Denhoff ER, Milliren CE, de Ferranti SD, et al. Factors associated with clinical research recruitment in a pediatric academic medical center-a web-based survey. PLOS ONE 2015;10:e0140768.

8 Daniels N. Fair process in patient selection for antiretroviral treatment in WHO's goal of 3 by 5 . Lancet 2005;366:169-71.

9 Persad G, Wertheimer A, Emanuel EJ. Principles for allocation of scarce medical interventions. Lancet 2009;373:423-31.

10 Cohen IG. Rationing legal services. J Leg Anal 2013;5:221-307.

11 Emanuel EJ, Wendler D, Grady C. What makes clinical research ethical? JAMA 2001;283:2701-11.

12 Chalmers I, Bracken MB, Djulbegovic B, et al. "How to increase value and reduce waste when research priorities are set. Lancet 2014;383:156-65.

13 Ioannidis JPA, Greenland S, Hlatky MA, et al. Increasing value and reducing waste in research design, conduct, and analysis. Lancet 2014;383:166-75.

14 Salman RA, Beller E, Kagan J, et al. Increasing value and reducing waste in biomedical research regulation and management. Lancet 2014;383:176-85.

15 Halpern SD, Karlawish JH, Berlin JA. The continuing unethical conduct of underpowered clinical trials. JAMA 2002;288:358-62.

16 Wertheimer A. Non-completion and informed consent. J Med Ethics 2014;40:127-30.

17 Appelabaum PS, Roth LH, Lidz CW, et al. False hopes and best data: consent to research and the therapeutic misconception. Hastings Cent Rep 1987:17:20-4. 